\title{
Preparation of CdSe Quantum Dots using kinetic growth method
}

\author{
Mekandan $\mathrm{M}^{*}$, Senthil Kumar $\mathrm{P}^{1}$, Sarala Ruby ${ }^{2}$, Ponraj vikaram ${ }^{3}$ \\ *,1,3, Department of mechanical, Anna University, Chennai - 25 \\ ${ }^{2}$ Department of Physics Vel Tech Technical university, Chennai - 54
}

\begin{abstract}
High quality quantum dots have been prepared with advances in the modern colloid chemistry. In the present study we have focused on preparation of CdSe quantum dots (QDs).CdSe prepared via colloidal synthesis in aqueous medium. CdSe QDs optical properties and size were characterized by ultraviolet-visible (UV-vis) absorption spectra, Photoluminescence spectra and DLS measurement. The result showed that the absorption spectra clearly indicate the absorption peak shift from $500 \mathrm{~nm}$ to $650 \mathrm{~nm}$ on increasing. The particle size average diameter of the CdSe quantum dot is reported as $13.36 \mathrm{~nm}$.
\end{abstract}

Keywords: Quantumdots, cadmium selenide, cadmium sulphide, ultraviolet-visible, Photoluminescence, dynamic light scattering.

\section{Introduction}

High quality quantum dots have been prepared with advances in the modern colloid chemistry ${ }^{[1]}$. Because of the interesting size-tailored photoluminescence and strong resistance against photochemical reaction in solution, quantum dots have been widely used as bio-labels ${ }^{[2-3]}$. However, it is still an interest of researchers to boost the quantum yield (defined as the ratio of emission to absorption photons) and, thus, reduce the number of the quantum dots needed in the experiment. The atoms on the surface of a quantum dot act as defects since they are not fully bonded. Many of these defects are removed by being passivated with organic surfactants during certain synthesis process. Although up to $10 \%$ quantum yield (at room temperature) of these capped dots has been reported ${ }^{[4]}$, it remains as a challenge to passivate both anionic and cationic sites on the surface with organic ligands. An alternative method to the use of organic molecules as surface passivation is to coat the surface with higher band gap inorganic semiconductor to form a core/shell structure. Not only are the dangling bonds of the core fixed by the shell, but the electron-hole pairs are more confined by the higher potential barriers as well ${ }^{[5]}$.

\section{Materials used}

\section{Experimental section}

The materials used were of the purest quality commercially available and used as received. Cadmium oxide (99.998\%) and Sulphur was purchased from Nice chemicals, Trioctylphosphine (95\%), Trioctylphosphineoxide (95\%), Octadecene and selenium powder (Se, 98.\%) were bought from Aldrich Chem. Co., and oleic acid was purchased from Aldrich Chemical Company.

\section{Preparation of Colloidal CdSe quantum dots}

CdSe nanocrystals are synthesized from $\mathrm{CdO}$ and elemental Se using a kinetic growth method ${ }^{[8-10]}$ where particle size depends on reaction time. A stock solution of Se precursor may be prepared ahead of time by combining $30 \mathrm{mg}$ of Se and $5 \mathrm{ml}$ of 1 -octadecene (tech., $90 \%$ ) in a $10-\mathrm{mL}$ round-bottom flask clamped over a stirrer hot plate. A syringe is used to measure $0.4 \mathrm{~mL}$ of trioctylphosphine from its Sure-Seal bottle to the same 10- $\mathrm{ml}$ flask. A magnetic stir bar is added and the solution is stirred for $30 \mathrm{~min}$. It is heated at $60 \mathrm{deg} \mathrm{C}$ to speed the dissolution of Se. The stock solution is stored at room temperature in a sealed container and has enough Se precursors for the preparations.

The Cd precursor is prepared by adding $65 \mathrm{mg}$ of $\mathrm{CdO}$ to a $250-\mathrm{mL}$ round-bottom flask clamped in a heating mantle. To the same flask $3 \mathrm{ml}$ of oleic acid and $50 \mathrm{ml}$ of octadecene are added. A thermometer capable of measuring $225^{\circ} \mathrm{C}$ is inserted, the temperature to which the flask is then heated. When the temperature reaches $225^{\circ} \mathrm{C}, 5 \mathrm{~mL}$ of the room-temperature selenium solution is transferred to the $225^{\circ} \mathrm{C}$ cadmium solution. Because the characteristics of the products depend on reaction time, one should begin timing when the selenium solution is added. A 9-inch Pasteur pipet is used to remove and quench approximately $2 \mathrm{ml}$ samples at frequent time intervals (0mins, $2 \mathrm{mins}$, $4 \mathrm{mins}$ and $6 \mathrm{mins}$ ) as quickly as possible in the beginning and when noticeable color change is detected at later times. The set of CdSe quantum dots are shown in Fig. The samples were pipetted out within six minutes and also we can see four different colors which indicate that different sizes of CdSe quantum dots. 


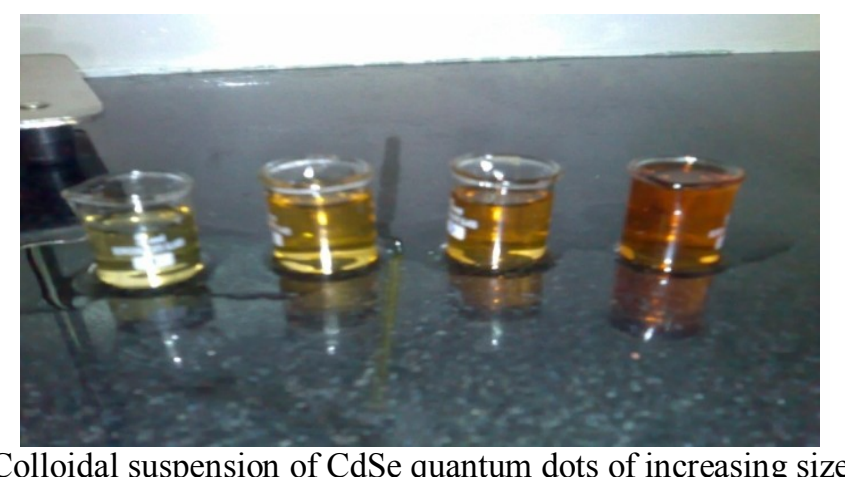

\section{Result and Discussion}

A major challenge in nanocrystal synthesis is the production of monodisperse particles. Monodisperse means the particles exhibit a relatively narrow size distribution, resulting in narrow absorbance and emission peaks. The growth of CdSe quantum dots could be identified by the color change in the UV-Vis spectra. DLS measurement also gives the size distribution and the average size of the prepared sample. The results obtained from the various measurements are discussed below. Prepared nanofibers were characterized by using Scanning Electron Microscope technique.

\section{Optical absorption}

\section{UV Spectrum for CdSe quantum dots}

The optical absorption wavelength was measured by using UV spectrometer and spectrum was taken for CdSe samples. The absorbance spectra of samples pipetted at different reaction times of $2 \mathrm{mins}$ and $6 \mathrm{mins}$ after injection of Se precursor is given in fig. The absorption spectrum shows striking excitonic features around $520 \mathrm{~nm}$ to $590 \mathrm{~nm}$ in the visible range. The absorption spectra clearly indicates that the absorption peak shift from $520 \mathrm{~nm}$ to $590 \mathrm{~nm}$ on increasing the particle size. It should be noted that such pronounced absorption spectrum predicts good crystallinity in the sample and also confirms that the material is a semiconductor.

\section{Light brownish (2mins):}

Size $\mathbf{- 5 4 3 . 1 n m ~ \& ~ a b s o r p t i o n ~ - 0 . 2 1 0 ~}$

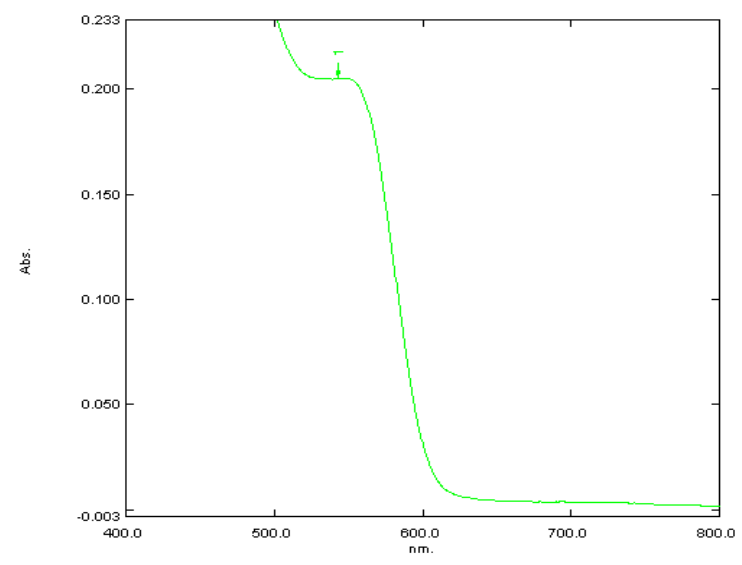

Brownish (6mins):

Size-577nm \& absorption-0.137 


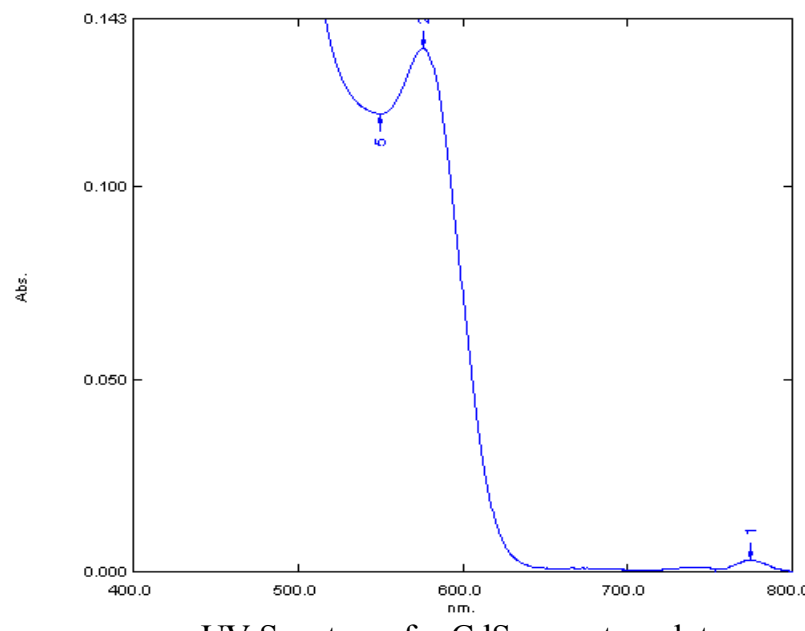

UV Spectrum for CdSe quantum dots

\section{DLS (Dynamic Light Scattering) Measurement}

Particle size for CdSe quantum dots

Dynamic Laser Light Scattering experiments were carried out for measuring the Size of the prepared CdSe quantum dots. The average Size Distribution Report of the CdSe quantum dots samples dispersed in an organic solvent-octadecene and pipetted at different reaction times of $2 \mathrm{mins}$ and $6 \mathrm{mins}$ after injection of Se precursor is shown in the below Fig4.2.1.The average diameters of the CdSe quantum dots are reported as $10.63 \mathrm{~nm}$ for light brownish color( $2 \mathrm{mis}$ ) and $13.88 \mathrm{~nm}$ for brownish color sample (6mins).

\section{Light brownish (2mins):}

\section{Brownish (6mins):}

$\begin{array}{rllll} & & \text { Diam. }(\mathrm{nm}) & \text { \% Intensity } & \text { Width }(\mathrm{nm}) \\ \text { Z-Average (r.nm): } 10.63 & \text { Peak 1: } & 7.445 & 62.7 & 4.198 \\ \text { Pdl: } 0.493 & \text { Peak 2: } & 1007 & 37.3 & 680.9 \\ \text { Intercept: } 0.103 & \text { Peak 3: } & 0.000 & 0.0 & 0.000\end{array}$

Result quality : Refer to quality report

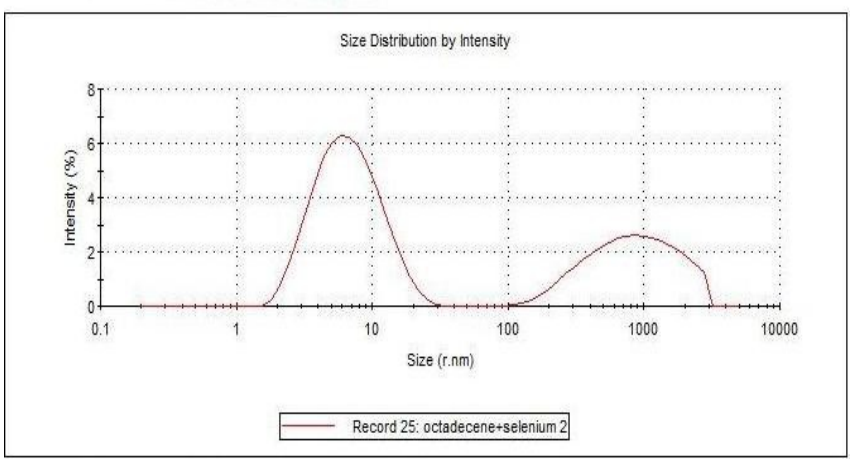

\begin{tabular}{|c|c|c|c|c|c|}
\hline & & & Diam. $(\mathrm{nm})$ & $\%$ Intensity & Width (nm) \\
\hline Z-Average $(r . n m)$; & 13.88 & Peak 1: & 6.020 & 54.4 & 4.587 \\
\hline Pdl: & 0.546 & Peak 2: & 1067 & 45.6 & 734.3 \\
\hline Intercept: & 0.104 & Peak 3: & 0.000 & 0.0 & 0.000 \\
\hline
\end{tabular}

Result quality : Refer to quality report

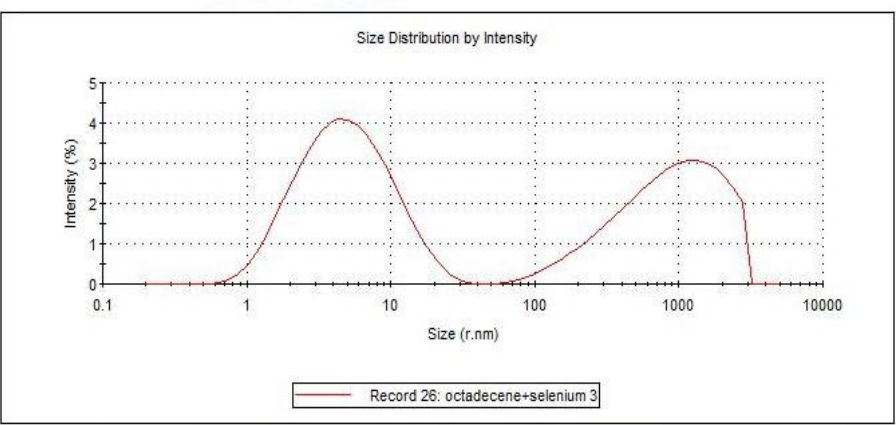

Average diameter of the CdSe quantum dots 


\section{Photoluminescence}

\section{PL Spectrum for CdSe QD'S}

The photoluminescence spectra emission maximum of CdSe is at $550 \mathrm{~nm}$, consistent with the red shifts visible in the absorption spectra. More importantly, the intensity of the emission maximum, which is normalized to the absorption spectrum, is considerably increased in the core/shell structure as compared to the parent materials (CdSe).

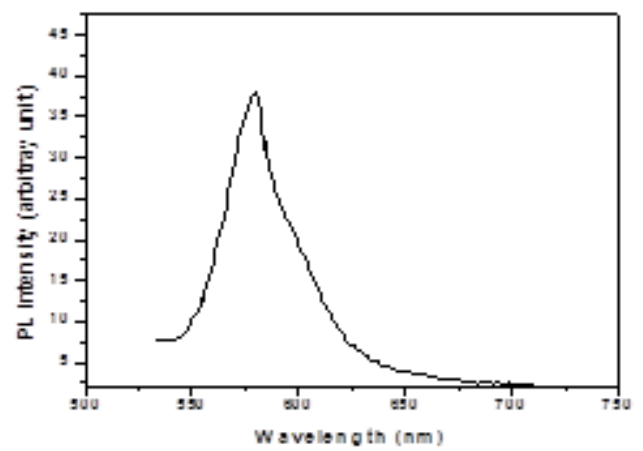

Photoluminescence spectrum $\left(\lambda_{\mathrm{ex}}=400 \mathrm{~nm}\right)$ of CdSe

\section{Conclusions}

CdSe has been synthesized from Cadmium oxide, Sulphur, Trioctylphosphine, Trioctylphosphineoxide, Octadecene, stearic acid and selenium powder precursors and their optical properties discussed.The absorption and emission spectra of CdSe structures has shown.. Particle Size Analyzer value shows prepared quantum dots are $10 \mathrm{~nm}$ to $14 \mathrm{~nm}$.

\section{Reference}

[1]. Murray, C. B., C. R. Kagan, et al. (2000). UAnnu. Rev. Mater. Sci.U 30: 545

[2]. Bruchez, M. P., M. Moronne, et al. (1998). UScienceU 281: 2013.

[3]. Chan, W. C. W. and S. Nie (1998). "Quantum Dot Bioconjugates for Ultrasensitive Nonisotopic Detection." UScienceU 281: 2016.

[4]. Michalet, X., F. F. Pinaud, et al. (2005). "Quantum dots for live cells, in vivo imaging and diagnostics." UScienceU 307: 583

[5]. Dabbousi, B. O., J. Rodriguez-Viejo, et al. (1997). "(CdSe)ZnS Core-Shell Quantum Dots: Synthesis and Characterization of a Size Series of Highly Luminescent Nanocrystallites." UJ Phys Chem BU 101: 9463.

[6]. Guo W., Li JJ., Wang YA., Peng X., Conjugation chemistry and bioapplications of semiconductor box nanocrystals prepared vis dendrimer bridging, Chem. Mater. 15 (2003) 3125-3133.

[7]. Elizabeth M, Boatman and George C.Lisensky (2005) 'A safer, Easier, Faster Synthesis for CdSe quantum dot Nanocrystals' journal of chemical education vol.82 pp.1697.

[8]. Jeaho park, Kwan Hyi Lee, Justin F.Galloway, and Peter C.Searson (2008) 'Synthesis of Cadmium selenide Quantum Dots from non-coordinating solvent: Growth Kinetics and particle size distribution' J.phys.chem. Vol.112 pp.17849-17854.

[9]. Seoudi R, Elokr M.M., Shabaka A.A., Sobhi.A. (2008) 'Synthesis, characterization and electrical properties studies of Cadmium Selenide nanoparticle' Physica B., Vol. 403 Pp.152-158.

[10]. Yang Z., Cingarapu S., Klabunde K.J., (2009) 'An unusual fluorescence evolution of cadmium selenide (CdSe) nanoparticles generated from a cadmium oxide/trioctylphosphine selenide/trioctylphosphin heterogeneous system', Chemical Physics Letters. 